\title{
A case of COVID-19 immediately after liver transplantation: Not only bad news
}

\author{
Mikel Prieto ${ }^{1,2}$, Mikel Gastaca ${ }^{1,2}$, Patricia Ruiz ${ }^{1}$, Alberto Ventoso ${ }^{1}$, Ibone Palomares ${ }^{1}$, \\ Regino José Rodríguez-Álvarez ${ }^{3}$, Patricia Salvador ${ }^{2,4}$, Javier Bustamante ${ }^{2,4}$, and Andrés Valdivieso ${ }^{1,2}$ \\ ${ }^{1}$ Hepatobiliary Surgery and Liver Transplant Unit, Cruces University Hospital, \\ ${ }^{2}$ Medicine Faculty, University of the Basque Country, ${ }^{3}$ Infectious Disease Unit, Cruces University Hospital, \\ ${ }^{4}$ Hepatology Unit, Cruces University Hospital, Bilbao, Spain
}

\begin{abstract}
COVID-19, the illness caused by the SARS-CoV-2 virus originated in December 2019 in Wuhan, China and has caused more 3,3 million cases and more than 230,000 deaths throughout the world, with 25,000 of them only in Spain, where the first case was diagnosed on January 31st, 2020. As COVID-19 is a "new" disease, we still do not have data on prognosis or treatment in transplant patients or on how to manage immunosuppression in this complex scenario. We present a case of COVID-19 diagnosed during the early postoperative period in a recipient whose liver transplantation was performed on late March during the lockdown in Spain, with donor and recipient previously negative rRT-PCR to SARS-CoV-2. In the first post-operative week the patient suffered COVID-19 pneumonia that was treated with immunosuppression minimization, oral Hydroxycloroquine and Azithromycin with favorable outcome. The patient was discharged on POD 21 without complications. To date, few early post-liver transplantation SARS-CoV-2 infected recipients have been published, but only one was an early postoperative infection. In our case the outcome was favorable, even though it was an early post -liver transplantation COVID-19 in a frail patient. (Ann Hepatobiliary Pancreat Surg 2020;24:314-318)
\end{abstract}

Key Words: Coronavirus; SARS-CoV-2; COVID-19; Transplantation; Liver

\section{INTRODUCTION}

The coronavirus disease-19 (COVID-19) originated in December 2019, in the city of Wuhan (Hubei, China) as an outbreak caused by a virus named "severe acute respiratory syndrome coronavirus 2" (SARS-CoV-2). The World Health Organization (WHO) declared COVID-19 a pandemic on March 11, 2020. As of May 3rd, 2020, the disease has been reported in 215 countries all over the world with 3,349,786 confirmed cases and over 238,628 deaths. ${ }^{1}$ In Spain, the first case was diagnosed on January 31 st, 2020, and to date, up to 217,466 cases have been diagnosed with 25,264 deaths reported. ${ }^{2}$ To date, Spain is the country with the second-highest number of confirmed COVID-19 cases. ${ }^{1}$

In general population, $20 \%$ of COVID-19 patients develop severe illness requiring hospital admission. Moreover,
$5 \%$ of patients need intensive care support, with a reported case fatality rate of $1-6 \%$ (11.6\% in Spain). ${ }^{2,3}$ Patients under chronic immunosuppression such as liver transplantation recipients (LT) may present atypical respiratory infections, difficult to distinguish from other postoperative infections. ${ }^{4}$ To date, few clinical experiences have been published on how SARS-CoV-2 affects immunosuppressed or transplant patients. ${ }^{5-7}$ On the other hand, there are some data among surgical patients with postoperative COVID-19 infection, $44.1 \%$ requiring Intensive Care Unit (ICU) with a mortality rate approaching $50 \%,{ }^{8}$ reflecting a much more higher ICU admission and mortality rates than non-surgical COVID19 patients. $^{3}$ Lei et al. ${ }^{8}$ suggest that surgery may accelerate and exacerbate disease progression of COVID-19.

To date, only one case of early postoperative COVID-19 disease has been described in LT. ${ }^{9}$ We present a case of

Received: May 6, 2020; Revised: June 2, 2020; Accepted: June 7, 2020

Corresponding author: Mikel Gastaca

Hepatobiliary Surgery and Liver Transplant Unit, Cruces University Hospital, Plaza de Cruces S/n, CP 48903, Bilbao, Spain Tel: +34-946002264, Fax: +34-946006372, E-mail: mikelgastaca@gmail.com

Copyright (C) 2020 by The Korean Association of Hepato-Biliary-Pancreatic Surgery

This is an Open Access article distributed under the terms of the Creative Commons Attribution Non-Commercial License (http://creativecommons.org/ censes/by-nc/4.0) which permits unrestricted non-commercial use, distribution, and reproduction in any medium, provided the original work is properly cited. Annals of Hepato-Biliary-Pancreatic Surgery - pISSN: 2508-5778 - elSSN: 2508-5859 
COVID-19 diagnosed during the early postoperative period in a recipient whose LT was performed at the end of March during the lockdown in Spain.

\section{CASE}

The liver recipient was a blood group A positive 52year-old male, with decompensated alcoholic cirrhosis and severe portal hypertension (MELD 20, encephalopathy, ascites, hepatorenal syndrome type II with an preoperative estimated glomerular filtration rate [eGFR] of $52 \mathrm{ml} / \mathrm{min}$ ), atrial fibrillation and a gastric by-pass surgery due to morbid obesity performed 7 years ago (actual body mass index was 35). He had been hospitalized until 2 days before the transplant due to recurrent liver complications and, despite not having fever or clinically evident infection, surveillance nasopharyngeal and oropharyngeal swabs for SARS-CoV-2 RT-PCT were obtained but resulted negative.

The donor was a blood group 0 positive, 47-year-old man without any previous disease who died from intracranial hemorrhage after a work-related accident. Following the current recommendations of the Spanish National Transplant Organization, ${ }^{10}$ the donor and next of kin were investigated for epidemiological risks or presence of clinical symptoms compatible with COVID-19. Even though they showed no specific risks or symptoms, a nasopharyngeal and oropharyngeal swab SARS-CoV-2 real-time reverse-transcriptase-polymerase-chain-reaction (rRT-PCR) was performed, which was negative. The liver was procured by standard methods and appeared healthy upon retrieval.

The cold ischemia time was 283 minutes, the warm ischemia time was 25 minutes and the total surgery time was 225 minutes. All anastomoses were done in a standard way following a piggyback technique. The patient required 1 unit of packed red blood cells administered intraoperatively.

The patient received Basiliximab for induction therapy (day 0 and day 4) combined with Mycophenolate Mofetil (MMF), 1 g/12 hours and glucocorticoids (Prednisone 20 mg per day), according to our protocol for patients with pretransplant renal dysfunction, with good postoperative graft function. ${ }^{11}$ On postoperative day (POD) 3, MMF was reduced to $500 \mathrm{mg} / 12$ hours due to pancytopenia. At that point, pancytopenia was thought to be to be related with surgery and his previous cirrhosis. The patient showed an uneventful initial course, so he was discharged from the ICU on the following day, without any infectious or respiratory symptoms. On POD 5 he presented with fever $\left(38^{\circ} \mathrm{C}\right)$, dyspnea, hypoxia (89\% on room air), and tachypnea without respiratory distress. Supplemental oxygen via nasal cannula (2 liters/minute), antipyretic (Paracetamol 1 g) and intravenous broad-spectrum antibiotic therapy (Meropenem $1 \mathrm{~g} / 8$ hours and Linezolid $600 \mathrm{mg} / 12$ hours) were started. Blood cultures were negative, and chest radiography showed diffuse bilateral infiltrates (Fig. 1). White blood cell count on peripheral blood was $4.9 \times 10^{3} / \mathrm{ml}$ with lymphopenia $\left(0.4 \times 10^{3} / \mathrm{ml}\right)$ and with a platelet count of $48 \times 10^{3} / \mathrm{ml}$, with a high ferritin $(783 \mathrm{ng} / \mathrm{ml})$ and high D-dimers $(16.200 \mathrm{ng} / \mathrm{ml})$ levels. The lactate dehydrogenase was normal (243 UI/L). Kidney (eGFR $>90 \mathrm{ml} / \mathrm{min}$ ) and liver graft function were normal. Considering the patient's immunosuppression and symptoms, and the pandemic state at the end of March, (at that time 335 patients with confirmed COVID-19 infection were hospitalized at Cruces University Hospital), ${ }^{12}$ a committee of transplant surgeons, hepatologist and infectious diseases specialists, decided to develop a secure pathway for all the contacts, and to repeat a nasopharyngeal/oropharyngeal swab SARSCoV-2 rRT-PCR to the patient, which was again negative. Two days after the chest radiography, we performed a CT on POD 7, but it did not show any characteristic images.

In spite of the patient's clinical improvement, and due to the high clinical suspicion, the secure pathway was maintained and a new nasopharyngeal/oropharyngeal swab for SARS-CoV-2 rRT-PCR was performed 48 hours later, that turned out positive. We contacted the donor center in order to retrieve new samples from the donor to per-

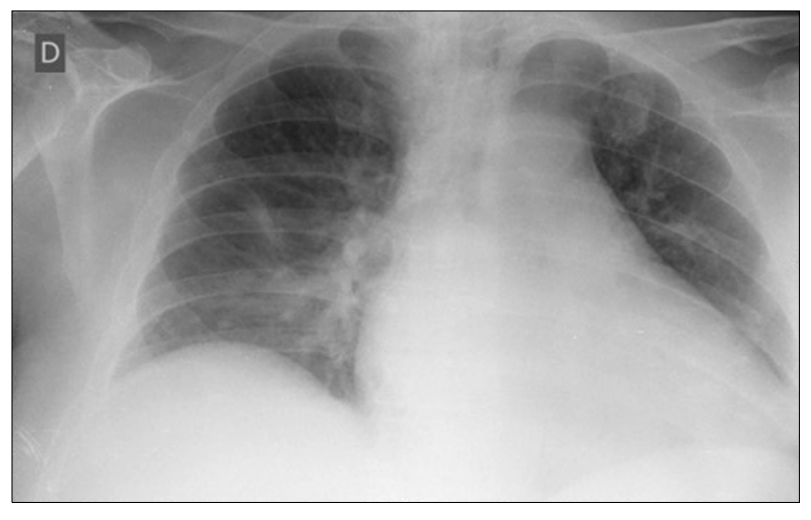

Fig. 1. Chest radiography showing diffuse bilateral infiltrates. 


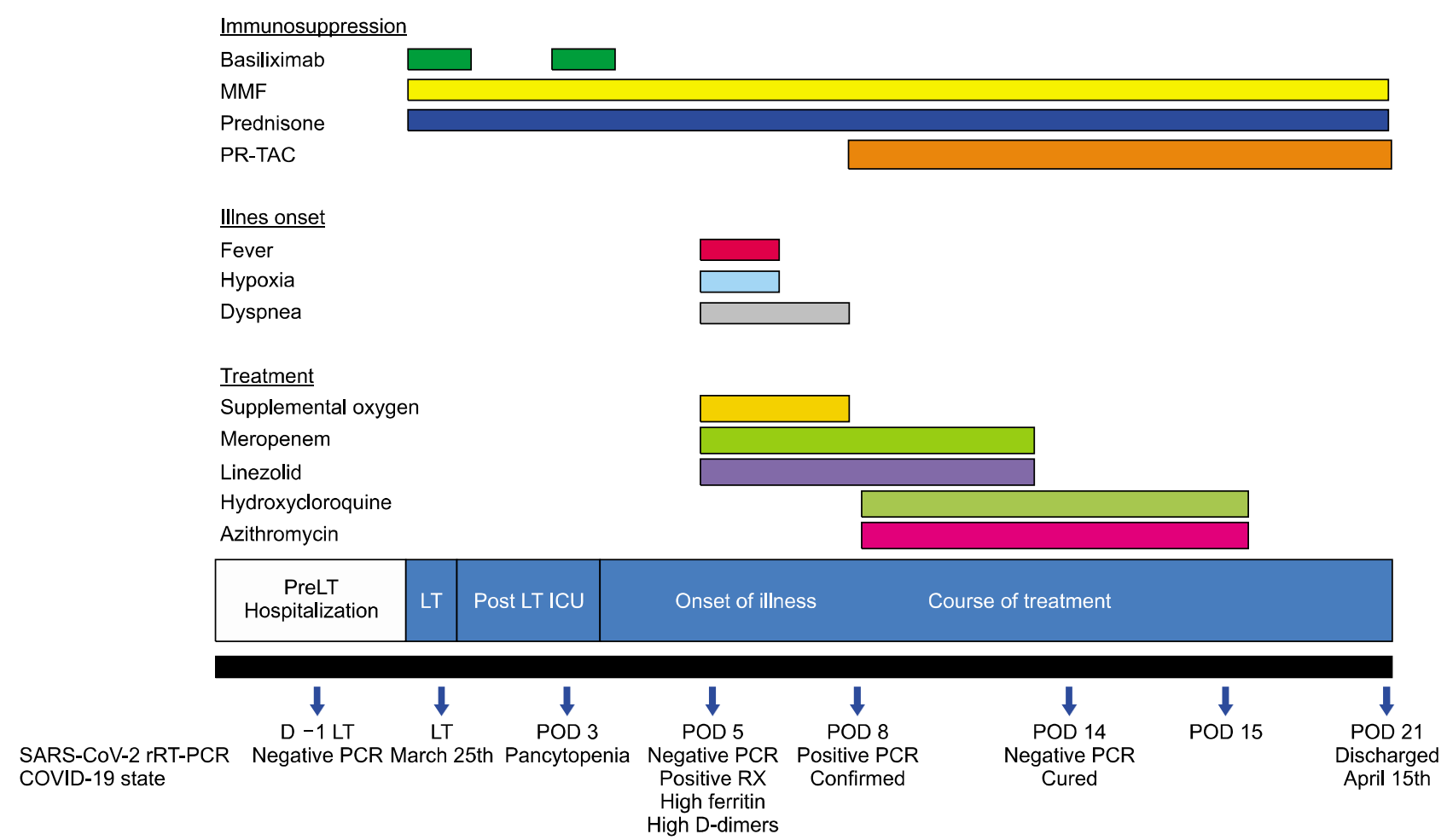

Fig. 2. Case report evolution. PR-TAC, prolonged released tacrolimus; D, day; LT, liver transplantation; POD, postoperative day.

form a SARS-CoV-2 serology, which were confirmed negative. Previous to start the treatment with oral Hydroxycloroquine and Azithromycin our patient was evaluated by the cardiologist, without contraindication. That day we decided to start treatment with oral Hydroxycloroquine (400 $\mathrm{mg} / 12$ hours for the first 24 hours and then $200 \mathrm{mg} / 12$ hours) and Azithromycin $(500 \mathrm{mg} / 24$ hours the first 24 hours and then $250 \mathrm{mg} / 24$ hours). ${ }^{13}$ Graft showed progressive cholestatic dysfunction (gGT 484 U/L, ALP 634 $\mathrm{U} / \mathrm{L}$ and bilirubin $7.2 \mathrm{mg} / \mathrm{dl}$ ) which was considered related to under immunosuppression so $0.05 \mathrm{mg} / \mathrm{kg} /$ day prolonged release Tacrolimus was introduced, in order to keep immunosuppression close to the lower range of our standard target levels for the initial posttransplant period (between 4-6 ng/ml). ${ }^{11}$ The patient was moved to a COVID-19 specific ward. Meropenem and Linezolid were discontinued after 8 days. Patient's general condition continued to improve. Nasopharyngeal/oropharyngeal swab for SARSCoV-2 rRT-PCR performed on POD 14 turned out negative. Lymphopenia $\left(2 \times 10^{3} / \mathrm{ml}\right)$, platelet count $\left(252 \times 10^{3} / \mathrm{ml}\right)$, ferritin $(469 \mathrm{ng} / \mathrm{ml})$ and D-Dimers $(4.240 \mathrm{ng} / \mathrm{ml})$ levels progressively normalized. Graft function quickly returned to normal after several adjustments of triple immuno- suppressive therapy. Hydroxycloroquine and Azithromycin were prescribed for 8 days and discontinued after the negative rRT-PCR. At the time the patient was diagnosed and treated only one negative SARS-CoV-2 rRT-PCR after treatment was the policy of the Spanish health ministry.

The patient was discharged on POD 21 without further complications (Fig. 2).

\section{DISCUSSION}

Severe acute respiratory syndrome coronavirus (SARS$\mathrm{CoV}$ ) and Middle East respiratory syndrome coronavirus (MERS-CoV) were reported in transplant recipients during prior outbreaks of these viruses. ${ }^{14}$ Although it is not yet known if immunosuppression is a risk factor for more severe disease, the transplant community eagerly awaits more studies in this patient population. ${ }^{6}$ The first two reported cases of solid organ transplantation (SOT) with COVID-19 were two heart transplant recipients from the city of Wuhan (Hubei, China). ${ }^{15}$ To date, few clinical cases of LT with COVID- $19^{8,9,16,17}$ and three series from Italy and USA with small number ${ }^{7,18,19}$ have been published and only one was an early infection just after LT. ${ }^{8}$ Recently, 
the first COVID-19 related death after LT has been reported. ${ }^{17}$

In our case, even though it was an early post-LT COVID19 in a frail patient, a favorable outcome was observed. As SARS-CoV-2 rt-PCR was negative for both donor and recipient, we decided to initiate intravenous broad-spectrum antibiotic therapy. The MMF dose had already been reduced due to pancytopenia. Due to the pandemic state at our country and center, and the high suspicion derived from the clinical status compatible with a stage IIa (moderate) pulmonary COVID-19 involvement, ${ }^{20}$ a new nasopharyngeal/oropharyngeal swab SARS-CoV-2 rRT-PCR was performed 48 hours later and COVID-19 was confirmed. Hydroxycloroquine and Azithromycin were immediately added while previous antibiotic therapy was progressively withdrawn.

Our patient showed symptoms on POD 5, in accordance with the findings by Lei et al. ${ }^{8}$ who suggest that patients may develop COVID-19 symptoms after general surgery procedures within a shorter period of time (between 2 and 6 days) than the conventional COVID- 19 patients. The secure pathway was maintained during all the postoperative period. The was no clinical evidence of SARS-CoV-2 infection in the transplantion group which includes surgeons, anesthetists, hepatologists, nurses and all manpower in direct contact with the donor or the recipient's family. Rt-PCR for SARS-CoV-2 has high sensitivity and specificity but these tests can show false negatives. Usually these are due to the sample being insufficient or unrepresentative, taken too early or too late in the course of the disease, or degraded during transport or handling. In negative cases in which suspicion or symptoms persist, it is recommended to repeat the Rt-PCR within a few days. ${ }^{20}$ The theory supporting a shorter incubation period may be difficult to apply here, because it is unknown whether the patient was in incubation period upon admission or if this was acquired during his hospital stay and showed two false negative Rt-PCR tests. It seems more clear that it is not a donor to recipient transmission of COVID-19 as the donor and next of kin were investigated for epidemiological risks or presence of clinical symptoms compatible without suspicion, the Rt-PCR and the serology were also negative.

It is known that in these initial stages, symptoms can be mild and non-specific; however, chest imaging may reveal bilateral infiltrates or ground glass opacities as, in our case. ${ }^{21}$ Currently, there is no strong evidence from controlled clinical trials to recommend a specific treatment for the SARS-CoV-2 coronavirus or the management of immunosuppression in LT in patients with confirmed COVID-19. The Spanish Society of Liver Transplantation guidelines recommend a reduction in $\mathrm{MMF}$ dose and maintenance of calcineurin inhibitor levels to the desired range in recipients with Stage I or IIa COVID-19. ${ }^{13}$ It is known that immunosuppressed patients have higher risk of developing infections; however, it is also possible that immunosuppressants could downregulate the deleterious inflammatory cascade characteristic of Stage III of this viral infection. ${ }^{6}$ Therefore, it may be possible that maintaining immunosuppressive treatment could help overcome the immunoreactive phase of the disease. In our case, we followed our standard immunosuppression policy for recipients with renal dysfunction: induction therapy, prednisone, MMF and delayed/reduced once-daily Tacrolimus. ${ }^{11}$ MMF was already reduced when COVID-19 was suspected, so we initially maintained Tacrolimus levels in the lower range; later Tacrolimus was progressively increased as needed, without clear impact on COVID19 evolution. Our local policy is to consider liver biopsy only when cholestatic dysfunction continues, after achieving tacrolimus levels within therapeutic range and other potential causes have been rule out. Regarding specific therapies for COVID-19 we followed the recommendations of the Spanish Society of Liver Transplantation and decided not to use Lopinavir/Ritonavir (very weak evidence of efficacy and significant drug interactions) but added oral Hydroxycloroquine and Azithromycin, which the risk/benefit ratio was considered acceptable despite the absence of clear evidence. ${ }^{13}$ Clinical trials, mostly for Stage III COVID-19 infection, are currently evaluating potential therapies including Remdesivir, which has been previously administered to Ebola virus patients, and others such as Tocilizumab. ${ }^{22}$

In Spain, universal screening (through nasopharyngeal and oropharyngeal rRT-PCR) is now mandatory for all donors across the country. In the Basque Country, with 193.04 COVID-19 cases per million population, ${ }^{2}$ we should balance the risks of postponing a livesaving transplant with the rationing of healthcare resources and the high risk of postoperative infection even when secure pathways are organized. In this pandemic scenario, a 
phased approach to decrease transplant activity has been recommended. ${ }^{23}$ In Spain a dramatic decrease in organ donation has been observed: from a mean of 15-18.6 donors per day in the first months of 2020 to a mean of 0.3-1.4 donors per day during the lockdown period. ${ }^{10}$

In conclusion, although liver recipients could be considered as potentially high-risk patients, favorable outcomes could be achieved even in case of postoperative COVID19 infection, provided that a quick diagnosis is made.

The fact that immunosuppressive state could be protective in severe COVID-19, as proposed by some authors, needs to be proved.

\section{ORCID}

Mikel Prieto: https://orcid.org/0000-0001-6662-4252

Mikel Gastaca: https://orcid.org/0000-0003-2771-9640

Patricia Ruiz: https://orcid.org/0000-0002-2598-0370

Alberto Ventoso: https://orcid.org/0000-0003-4635-8545

Ibone Palomares: https://orcid.org/0000-0002-0002-7436

Regino José Rodríguez-Álvarez:

$$
\text { https://orcid.org/0000-0001-8779-412X }
$$

Patricia Salvador: https://orcid.org/0000-0001-7741-9465

Javier Bustamante: https://orcid.org/0000-0002-5280-3038

Andrés Valdivieso: https://orcid.org/0000-0002-2614-3670

\section{REFERENCES}

1. World Health Organization. Coronavirus disease (COVID-19) outbreak [Internet]. Geneva: World Health Organization; 2020 [cited 2020 May 3]. Available from: https://www.who.int/emergencies/ diseases/novel-coronavirus-2019.

2. Spanish Ministry of Health. COVID-19 disease [Internet]. Paseo del Prado: Ministerio de Sanidad; 2020 [cited 2020 May 3]. Available from: https://www.mscbs.gob.es/en/profesionales/saludPublica/ ccayes/alertasActual/nCov-China/documentos/Actualizacion 67_COVID-19.pdf.

3. Wu Z, McGoogan JM. Characteristics of and important lessons from the coronavirus disease 2019 (COVID-19) outbreak in China: summary of a report of 72314 cases from the Chinese Center for Disease Control and Prevention. JAMA 2020;323:1239-1242.

4. Shelhamer JH, Toews GB, Masur H, Suffredini AF, Pizzo PA, Walsh TJ, et al. NIH conference. Respiratory disease in the immunosuppressed patient. Ann Intern Med 1992;117:415-431.

5. Michaels MG, La Hoz RM, Danziger-Isakov L, Blumberg EA, Kumar D, Green M, et al. Coronavirus disease 2019: implications of emerging infections for transplantation. Am J Transplant 2020;20:1768-1772.

6. D'Antiga L. Coronaviruses and immunosuppressed patients: the facts during the third epidemic. Liver Transpl 2020;26:832-834.

7. Pereira MR, Mohan S, Cohen DJ, Husain SA, Dube GK, Ratner
LE, et al. COVID-19 in solid organ transplant recipients: Initial report from the US epicenter. Am J Transplant 2020;20:18001808.

8. Lei S, Jiang F, Su W, Chen C, Chen J, Mei W, et al. Clinical characteristics and outcomes of patients undergoing surgeries during the incubation period of COVID-19 infection. Version 2. EClinicalMedicine 2020. doi: 10.1016/j.eclinm.2020.100331. [in press]

9. Qin J, Wang H, Qin X, Zhang P, Zhu L, Cai J, et al. Perioperative presentation of COVID-19 disease in a liver transplant recipient. Hepatology 2020. doi: 10.1002/hep.31257. [in press]

10. National Transplant Organization. Spanish recommendations to manage organ donation and transplantation regarding the infection associated with the new coronavirus (SARS-CoV-2) producer of COVID-19 [Internet]. Paseo del Prado: Ministerio de Sanidad; 2020 [cited 2020 Apr 16]. Available from: http://www.ont. es/infesp/RecomendacionesParaProfesionales/Spanish\%20Recom mendations \%20on\%20Organ\%20Donation\%20and\%20Transplan tation\%20COVID-19\%20\%20ONT.pdf.

11. Gastaca M, Prieto M, Palomares I, Bustamante J, Fernandez JR, Ruiz $\mathrm{P}$, et al. Long-term outcomes of liver transplantation in patients with pretransplant renal dysfunction treated with induction therapy and delayed reduced de novo once-daily tacrolimus. Transplant Proc 2020;52:1489-1492.

12. Osakidetza. Basque Health Department [Internet]. San Juan: Departamento de Salud; 2020 [cited 2020 Mar 30]. Available from: http://www.euskadi.eus/contenidos/informacion/boletin_corona virus/es def/adjuntos/30 marzo Boletin.pdf.

13. Spanish Society of Liver Transplant. Recommendations of the SSLT against COVID-19 in liver transplantation [Internet]. [cited 2020 Apr 13]. Available from: https://www.sethepatico.org/docs/2020/ Reco mend_IMS_COVID_TH.pdf.

14. AlGhamdi M, Mushtaq F, Awn N, Shalhoub S. MERS CoV infection in two renal transplant recipients: case report. Am J Transplant 2015;15:1101-1104.

15. Li F, Cai J, Dong N. First cases of COVID-19 in heart transplantation from China. J Heart Lung Transplant 2020;39:496-497.

16. Liu B, Wang Y, Zhao Y, Shi H, Zeng F, Chen Z. Successful treatment of severe COVID-19 pneumonia in a liver transplant recipient. Am J Transplant 2020;20:1891-1895.

17. Huang JF, Zheng KI, George J, Gao HN, Wei RN, Yan HD, et al. Fatal outcome in a liver transplant recipient with COVID-19. Am J Transplant 2020;20:1907-1910.

18. Bhoori S, Rossi RE, Citterio D, Mazzaferro V. COVID-19 in long-term liver transplant patients: preliminary experience from an Italian transplant centre in Lombardy. Lancet Gastroenterol Hepatol 2020;5:532-533.

19. Webb GJ, Moon AM, Barnes E, Barritt AS, Marjot T. Determining risk factors for mortality in liver transplant patients with COVID-19. Lancet Gastroenterol Hepatol 2020;5:643-644.

20. Fang $\mathrm{Y}$, Zhang $\mathrm{H}$, Xie J, Lin M, Ying L, Pang P, et al. Sensitivity of chest CT for COVID-19: comparison to RT-PCR. Radiology 2020;296:E115-E117.

21. Siddiqi HK, Mehra MR. COVID-19 illness in native and immunosuppressed states: a clinical-therapeutic staging proposal. J Heart Lung Transplant 2020;39:405-407.

22. Costanzo M, De Giglio MAR, Roviello GN. SARS-CoV-2: recent reports on antiviral therapies based on lopinavir/ritonavir, darunavir/umifenovir, hydroxychloroquine, remdesivir, Favipiravir and other drugs for the treatment of the new coronavirus. Curr Med Chem 2020;27:4536-4541.

23. Kumar D, Manuel O, Natori Y, Egawa H, Grossi P, Han SH, et al. COVID-19: a global transplant perspective on successfully navigating a pandemic. Am J Transplant 2020;20:1773-1779. 